\title{
Journal of \\ Self-assembly of latex particles for the creation of nanostructures with tunable plasmonic properties
}

\author{
Piotr Patoka and Michael Giersig* \\ Received 3rd May 2011, Accepted 11th July 2011 \\ DOI: $10.1039 / \mathrm{c} 1 \mathrm{jm} 11936 \mathrm{~b}$
}

In this short review we are discussing the creation of two dimensional nanostructures based on selfassembly of latex particles. Furthermore we are showing that optical properties of these structures can be controlled with their morphology and materials used for their preparation. Two representative structures, namely two dimensional arrays of triangles and holes are discussed in detail, starting with the preparatory step, followed by the structural and optical characterization, as well as the theoretical explanation of the plasmonic properties.

\section{Introduction}

The general direction of self-organization of nanoparticles has stimulated our research for creating new nanostructures with extraordinary optical properties. In this review we will briefly

Institut für Experimentalphysik, Freie Universität Berlin, Arnimallee 14, 14195 Berlin, Germany. E-mail: giersig@physik.fu-berlin.de discuss the nanosphere lithography and the possibility of creating new two-dimensionally organized nanostructures.

We use self-assembly of latex nanospheres for the creation of a colloidal monolayer, which serves as a mask during the material deposition process. The modification of the mask and change of evaporation geometry enables us to create variously shaped nanopatterns. Selection of a proper material for the evaporation process, i.e., gold or silver, which exhibits plasmonic responses in

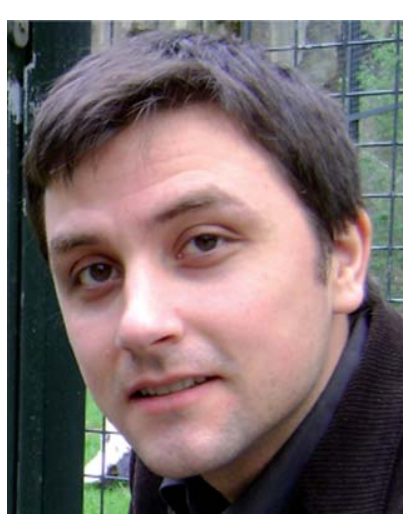

Piotr Patoka
Piotr Patoka, Freie Universität Berlin, Department Physics. He has joined Prof. Michael Giersig group in 2006. In 2007 he received his Master of Science degree at University of Wroclaw, Poland. Since 2007 he continued his study as a PhD student under supervision of Prof. Michael Giersig and finished in June 2011. His primary research area is fabrication of various shaped structures created by the means of shadow nanosphere lithography and studying their structural as well as optical properties with main focus on plasmonics.

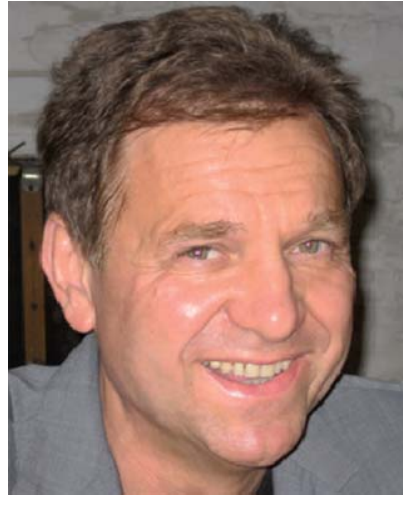

Michael Giersig
Professor Michael Giersig, Freie Universität Berlin, Department Physics. The goals of Michael Giersig Research lie in the preparation of nanometersized semiconductor, metallic, and magnetic particles, followed by the creation of periodically ordered nanostructures (1-D, 3-D) based on single nanoparticles. An important factor in this context so far has been the design and fabrication of nanocomponents with/displaying new functionalities and characteristics for the improvement of existing materials; including photonic materials, conductive materials, polymers and composites. With this concept of nanotechnology in mind, we aim at developing innovative products and application options in electronics and biomedicine, based solely on nanoscale technology. Giersig has published over 216 internationally refereed publications blanket physics, chemistry, materials science, biochemistry, medicine, nanotechnology and engineering. His work has been over 12100 times quoted in the ISI Index at an average of over 55 citations per publication. 
the visible range considerably broadens the application of such nanostructures. Here, in this work, we would like to show how one can control these plasmonic responses through the change of a shape of the structures, material, and position of the samples during the characterization.

\section{Self-assembled mask creation and modification}

\subsection{Colloidal mask preparation}

The basis of NSL is a self-assembly of a hexagonal close-packed (hcp) monolayer of polystyrene (PS) latex spheres. ${ }^{1-10}$ Since the production of such spheres is well-established on the market, there is an enormous possibility of choosing between various sizes and chemical stabilizations of PS micro and sub-micron spheres, also known as PS latex. Polystyrene spheres used in this work range between 440 and $1710 \mathrm{~nm}$ in diameter. Usually latex beads are shipped in water solutions with 4 or $8 \%$ concentrations of solid which must be increased. For this purpose, centrifugation (Laborfuge 400R, Heraeus) at 5000-6000 rpm for 15-30 min (depending on sphere size), followed by redispersion in an ethanolic/water polystyrene solution to a concentration of $30 \%$ was prepared and thoroughly mixed in an ultrasonic bath (Sonorex, $40 \mathrm{~W}$ ) for $30 \mathrm{~min}$. The prepared solution was slowly applied on a water surface using a glass pipette. All PS monolayers were assembled inside a $20 \mathrm{~cm}$ diameter Petri dish [Fig. 1a]. ${ }^{11}$ During the preparation one has to take into account the amount of the applied solution onto the water surface, leaving some place for stress relaxation and to avoid the formation of cracks in the lattice during the following steps of the preparation.

At this stage the monolayer had crystals of $2 \mathrm{~cm}^{2}$ in size, with very irregular shapes. To promote the growth of large crystals, the beaker was gently tilted to produce waves on the surface. After this treatment, crystals of about $50 \mathrm{~cm}^{2}$ were created showing clear diffraction colors, which are an indication of the formation of monocrystals. By adding a small amount of surfactant [Fig. 1b] a hexagonal close-packed hcp layer of spheres was formed and then anchored with one side to the edge of a Petri dish. Next, the desired substrates needed to be prepared. The samples were cut from wafers into $10 \times 10 \mathrm{~mm}^{2}$ pieces and pre-cleaned by $5 \mathrm{~min}$ ultrasonication and rinsing with 2-propanol. Following this, the substrates were submerged for $30-60 \mathrm{~min}$ in a standard cleaning solution $(\mathrm{SC} 1)^{12}$ of $7 \%$ $\mathrm{NH}_{4} \mathrm{OH} / 30 \% \mathrm{H}_{2} \mathrm{O}_{2} / \mathrm{Milli}-\mathrm{Q}$ water (vol. ratio $1: 1: 5$ ) at $80{ }^{\circ} \mathrm{C}$,

a)

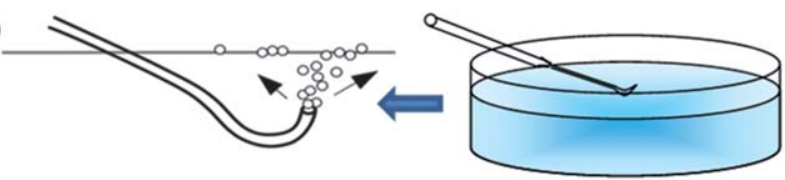

b)

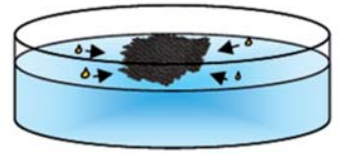

c)

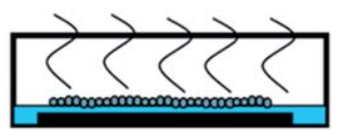

Fig. 1 Colloidal mask preparation: (a) applying of the solution to the water surface, (b) addition of surfactant-close packing and (c) deposition of a monolayer on a substrate. then rinsed thoroughly in Milli-Q water. The substrates were put into water at the opposite side of the anchored mask to avoid its destruction, placed at the bottom of the beaker, and aligned under the PS monolayer. Most of the water from the Petri's dish was pumped out slowly (about $50 \mathrm{ml} \mathrm{min}^{-1}$ ) with a membrane pump until the mask nearly touched the surface of the substrate. Finally, the monolayer was deposited onto the substrate by slow evaporation of the rest of the water [Fig. 1c]. The next step in the sample preparation was the transfer of the mask onto the substrate. The final result of this procedure is presented in Fig. 2a. Depending on the mask quality, one or more iridescent colors were observed on the surface of the substrates. For more detailed studies the samples were routinely examined with scanning electron microscopy (SEM). In this case SEM-Supra 55 with Gemini ${ }^{\circledR}$ column, from LEO Electron Microscopy Group was used to image the surface of the samples over large areas, which is very helpful to estimate the quality of the final structure. The images were acquired at an acceleration voltage of $2-6 \mathrm{kV}$ for nonconductive, and 3-25 kV for conductive substrates, using an in-lens detector. Fig. 2 b shows exemplary result of the imaging of masks prepared with latex spheres which were $440 \mathrm{~nm}$ in diameter. One can see that the result of the colloidal mask preparation technique is a monolayer of latex spheres assembled into a lithographic mask and transferred to a solid substrate.

These colloidal masks can be modified by reactive ion etching (RIE), which enhances the NSL to produce structures of different morphologies. The details about this process are presented in the following section.

\subsection{Mask modification-reactive ion etching}

The PS-masks deposited on the substrates can be modified in a post treatment etching process in which the diameter of the spheres can be reduced in a controllable way to a desired size. For this reason, previously prepared samples were introduced to the RIE. The etching is a very homogeneous process but to assure a good result one needs to fix the latex spheres on the substrates to avoid any movement during further processing.

To ensure this, samples covered with latex particles were put on a heating plate at $105{ }^{\circ} \mathrm{C}$ (temperature is slightly above the glass transition temperature of the PS particles) for $c a$.

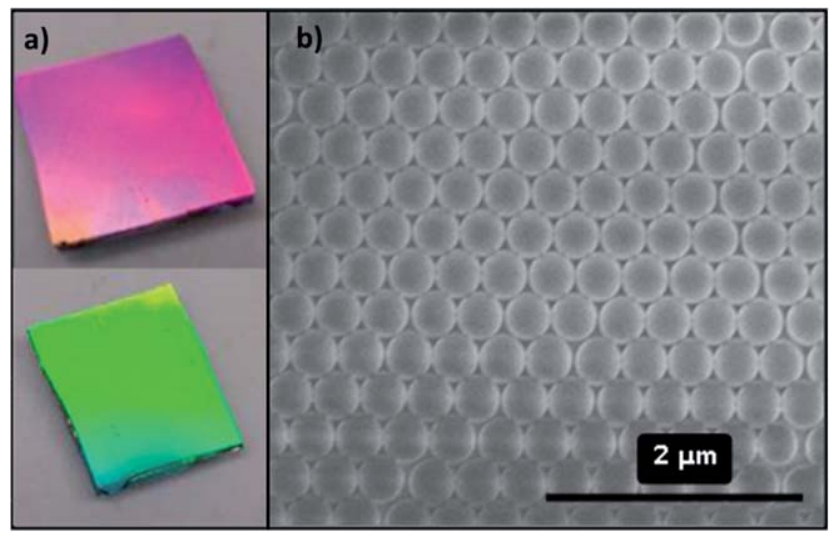

Fig. 2 PS-masks on a silicone substrates: (a) photographs taken under two different angles, (b) SEM image of hcp monolayer. 


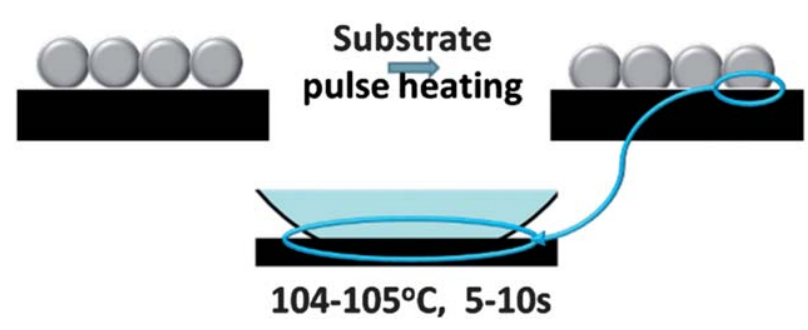

Fig. 3 Fixing of the PS mask to a substrate.

$10 \mathrm{~s}$ [Fig. 3]. This duration was derived experimentally and depends much on the thickness and heat conductivity of the substrate. The RIE process utilizes dissociation and ionization of neutral gas in an alternating electrical field and consists of two simultaneous sub-processes: first ions that were accelerated in an electric field collide with PS molecules and fracture them into radicals. This directional mechanical bombardment is called physical etching. Secondly, at the same time as the physical etching, an isotropic chemical etching caused by highly reactive neutral radicals diffusing to all surfaces proceeds. ${ }^{13}$ During the RIE process, the side products of both etching mechanisms are constantly removed from the reaction chamber by a vacuum pump [Fig. 4].

The reactivity ratio: isotropic/anisotropic of sub-processes can be controlled with a content of the procedure in which the most important parameters are the gas composition, plasma energy, pressure, and flow rate of reactive gas as well as the processing time. All details on RIE processes can be found in ref. 14. All samples that needed a RIE mask modification are processed using a Mulitplex Atmospheric Cassette System (MACS) from Surface Technology Systems with the electrodes in the reactor in parallel-plate configurations. PS spheres are etched using a $60 \mathrm{~W}$ RF power plasma at a pressure of $8 \mathrm{~Pa}$, while etching ioncompositions include argon and oxygen. The amount of applied gases can vary depending on the size of the used spheres. For smaller spheres (around $500 \mathrm{~nm}$ in diameter) one should use more oxygen, which instigates more aggressive etching. Such a procedure requires a shorter process time. In contrary, for larger particles one should extend the etching duration and reduce the amount of oxygen gas. The process time for this work varied from 0 to $65 \mathrm{~s}$. The results of the etching of the PS sphere masks are presented in Fig. $5 .{ }^{15}$ PS $440 \mathrm{~nm}$ mask is deposited on
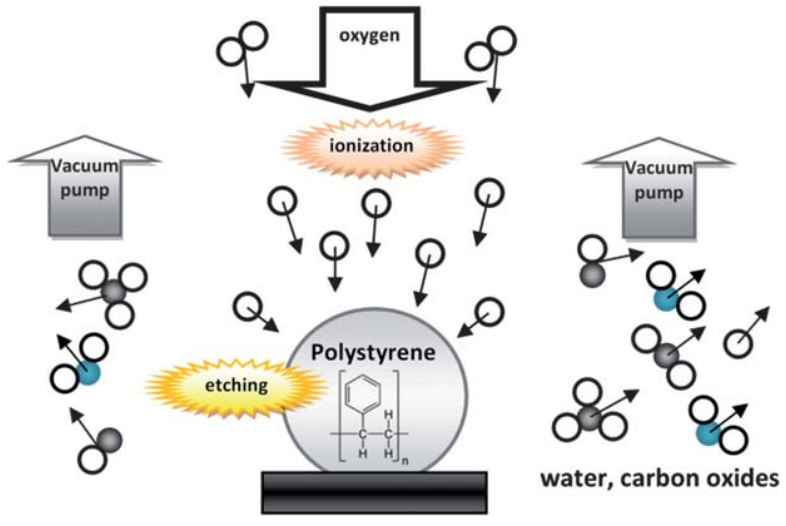

Fig. 4 Reactive ion etching.

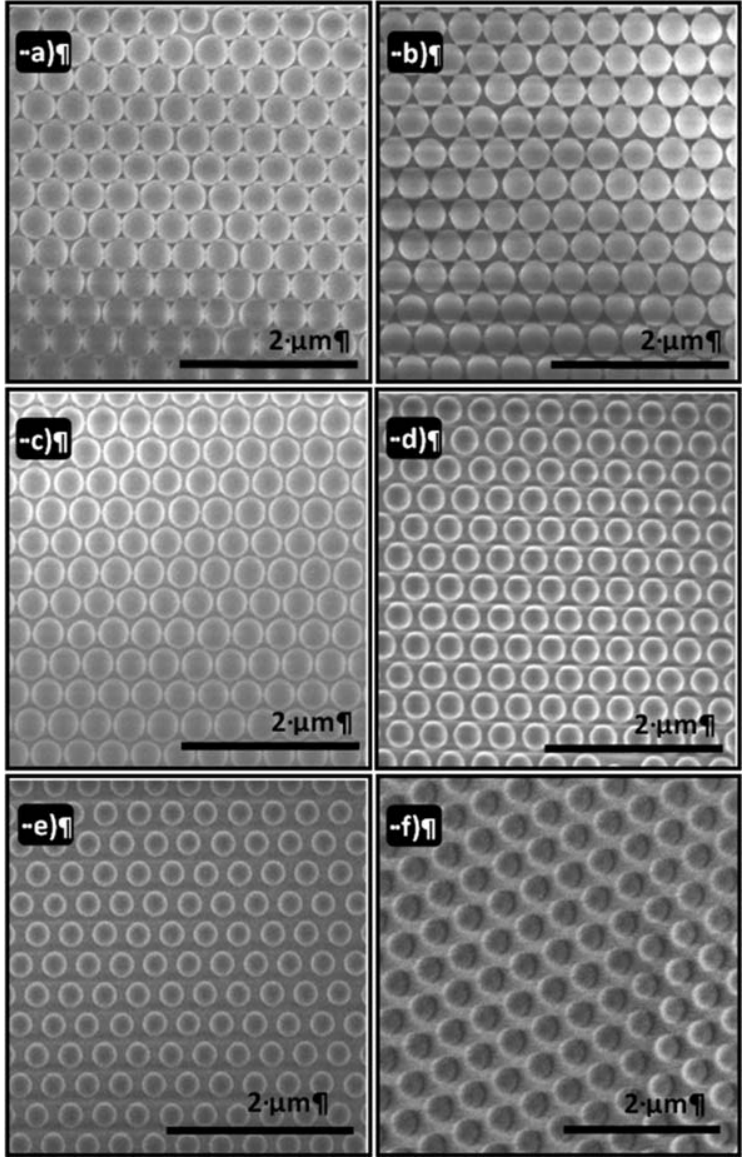

Fig. 5 SEM images of etched PS-masks. RIE process duration: (a) $0 \mathrm{~s}$, (b) $20 \mathrm{~s}$, (c) $35 \mathrm{~s}$, (d) $40 \mathrm{~s}$, (e) $45 \mathrm{~s}$ and (f) $50 \mathrm{~s}$.

$\alpha-\mathrm{Al}_{2} \mathrm{O}_{3}$ substrate, annealed for $5 \mathrm{~s}$ at $105{ }^{\circ} \mathrm{C}$ and etched in periods of 20-50 s using $\mathrm{Ar} / \mathrm{O}_{2}(10 / 35 \mathrm{sccm})$ plasma, driven at $60 \mathrm{~W}$ and $8 \mathrm{~Pa}$. The samples were characterized by SEM [Fig. 5], showing the reduced size of the spheres while the etching time was extended. Small differences in processing time $(5 \mathrm{~s})$ led to noticeable changes in the beads' diameter, starting from not processed $440 \mathrm{~nm}$ PS-masks [Fig. 5a], with the resulting diameters for process durations of 20,35, 40, 45 and 50 s being 390 , $370,340,300$ and $285 \mathrm{~nm}$ respectively [Fig. 5b-f].

The prepared colloidal masks transferred to the solid substrates can act as a shadow mask for the material deposition process which is the basis of the NSL. Since the metal deposition process is very crucial for the structure development, a brief introduction to the evaporation setup will be given.

\subsection{Material deposition}

All evaporation processes were done using a modified e-beam evaporator (BAK 640, Unaxis). Since the machine is a highthroughput system, it has an umbrella shaped holder for $245^{\prime \prime}$ wafers. Such a setup could not provide the necessary sample position control, therefore a separated holder is designed for shadow nanosphere lithography (SNSL) purposes which gives the necessary degrees of freedom to position the sample, of which the most crucial is the incident angle $\theta$ for the fabrication process. 
The distance between sample and evaporation source was $50 \mathrm{~cm}$. Therefore it was safe to consider the trajectories of atoms that hit the sample as parallel to each other. During all deposition processes the base pressure in the chamber was lower than $10^{-7} \mathrm{mbar}$, while the material deposition rate was kept constant at $0.5 \frac{\AA}{\mathrm{s}}$. The thickness of the deposited material was controlled by a built-in quartz-crystal microbalance (QCM).

\subsection{Mask lift off}

After the material was transferred to the substrate, the PS mask could be removed in two ways depending on the thickness of the deposited film: (1) film thickness below $50 \mathrm{~nm}$-adsorption of PS spheres to an adhesive tape (Tesa film), then peeling it from the substrate; (2) film thickness above $50 \mathrm{~nm}$-lift-off treatment is based on ultrasonication of the samples in tetrahydrofuran (THF), 1-methyl 2-pyrrolidone (NMP), or toluene (in some cases acetone was a sufficient solvent). Depending on the film thickness and type of the structure, ultrasonication took from $30 \mathrm{~s}$ up to $1 \mathrm{~h}$. The temperature of the dissolving medium was increased up to about $70{ }^{\circ} \mathrm{C}$ to improve cleaning. After THF, NMP or toluene treatment, the sample was rinsed several times with 2-propanol or acetone, then in Mill-Q water, and dried in a stream of argon.

\section{Creation of different shapes of structures}

\subsection{Preparation of triangles}

The simplest nanostructures that one can create with this lithographic method, is an array of triangular shaped nanoislands. The production procedure consists of the use of a non modified colloidal crystal deposited on a substrate as a mask for a material deposition process. During material evaporation the sample is set normal to the evaporation directions, i.e., there is no tilt between material source and sample $\left(\theta=0^{\circ}\right)$. The material evaporation and mask lift off process resulted in an array of triangular shaped particles. Atomic force microscopy was used to characterize the dimensions of the nanostructures. The AFM measurements were performed with Dimension 3100 with Nanoscope IV controller from Veeco. The samples were scanned in tapping mode using silicon tips, which had a nominal resonant frequency of 150 and $300 \mathrm{kHz}$ respectively. Both kinds of AFM sensors had a tip radius of less than $10 \mathrm{~nm}$ according to the data provided by the manufacturer (Veeco Probes). The exemplary results of the sample preparation are shown in Fig. $6 .{ }^{15}$

Here, a gallery of arrays of different sized triangles is depicted. The initial spheres used for fabrication of triangles were: (a) $380 \mathrm{~nm}$, (b) $540 \mathrm{~nm}$, (c) $980 \mathrm{~nm}$ (d) and (e) $1710 \mathrm{~nm}$ in diameter. It is clear that triangular particles follow the geometrical dependencies derived from the lithographic masks, thus their morphology can be easily deducted from the mask parameters. The sizes of these particles are proportionally to the diameter $\mathrm{D}_{0}$ of the spheres used to build the mask.

\subsection{Preparation of holes}

The etching process is increasing the area of the void spaces between the polystyrene spheres and causes the contact areas between them to split open. It can be used for the production of

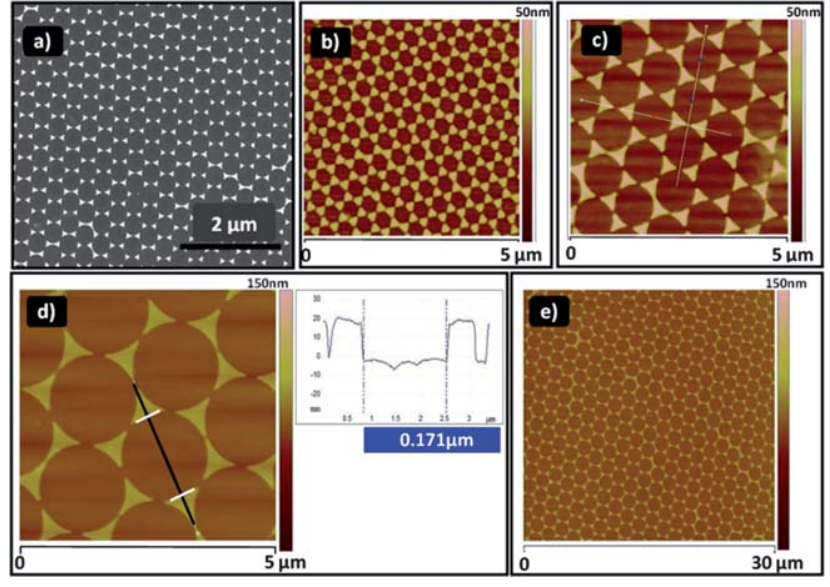

Fig. 6 Nanotriangles arrays fabricated with different colloidal mask size: (a) $380 \mathrm{~nm}$, (b) $540 \mathrm{~nm}$, (c) $980 \mathrm{~nm}$, (d) and (e) $1710 \mathrm{~nm}$, respectively.

arrays of subwavelength holes in metallic films. In short, as in the case of triangular shaped structures formed from PS-spheres with a diameter of $440 \mathrm{~nm}$, monolayer was deposited on sapphire chips. As we know, this close-packed structure prohibits the growth of an array of particles. Thus the diameter of the PSspheres had been reduced by means of reactive ion etching. Samples were modified in a RIE chamber using the following constant parameters: $\mathrm{Ar}=10 \mathrm{sccm}, \mathrm{O}_{2}=35 \mathrm{sccm}, \mathrm{P}=60 \mathrm{~W}, \mathrm{p}=$ $8 \mathrm{~Pa}$, while the process duration was varied between 20 and $50 \mathrm{~s}$. Afterwards, the obtained structure was used as a mask in the metal evaporation process. The deposition of $50 \mathrm{~nm}$ of material (e.g., aluminum) onto the samples took place under the same conditions as in the case of formation of the nanotriangles: the evaporation angle was kept at $\theta=0^{\circ}$. Finally, all samples were cleaned with the standard mask lift-off procedure.

The samples were routinely characterized under a scanning electron microscope, which confirmed the formation of a large area of defect-free arrays of subwavelength holes [Fig. 7a] with a high quality long range order [Fig. 7b]. Secondly the investigations of series of samples revealed that applying different etching times resulted in decreasing hole diameters in structures. The consequence of the RIE process duration set to $20,30,40$, and $50 \mathrm{~s}$ and evaporation of $50 \mathrm{~nm}$ of $\mathrm{Al}$ were holes with diameters of $385,340,325$, and $254 \mathrm{~nm}$, respectively, as is shown in Fig. 8.

\section{Tunable plasmonic properties}

Investigations of the optical properties were carried out with an UV-Vis-NIR spectrometer (Varian, Carry 5000) between 200 and $3000 \mathrm{~nm}$. The spectrometer is equipped with a home built sample holder, which enabled to change the incidence angle $\theta_{\text {inc }}$ [Fig. 9a].

In addition near-field optical investigations were performed using a combined AFM/scanning near-field optical microscope (SNOM; Mutliview 1000, Nanonics Imaging Ltd). A schematic of the SNOM setup is shown on Fig. 9b. A He-Cd laser set for operation at wavelength of $442 \mathrm{~nm}$ is used as an illumination source. In this setup light passes through a half-wave plate and a polarizer, this enables control of the intensity and the 


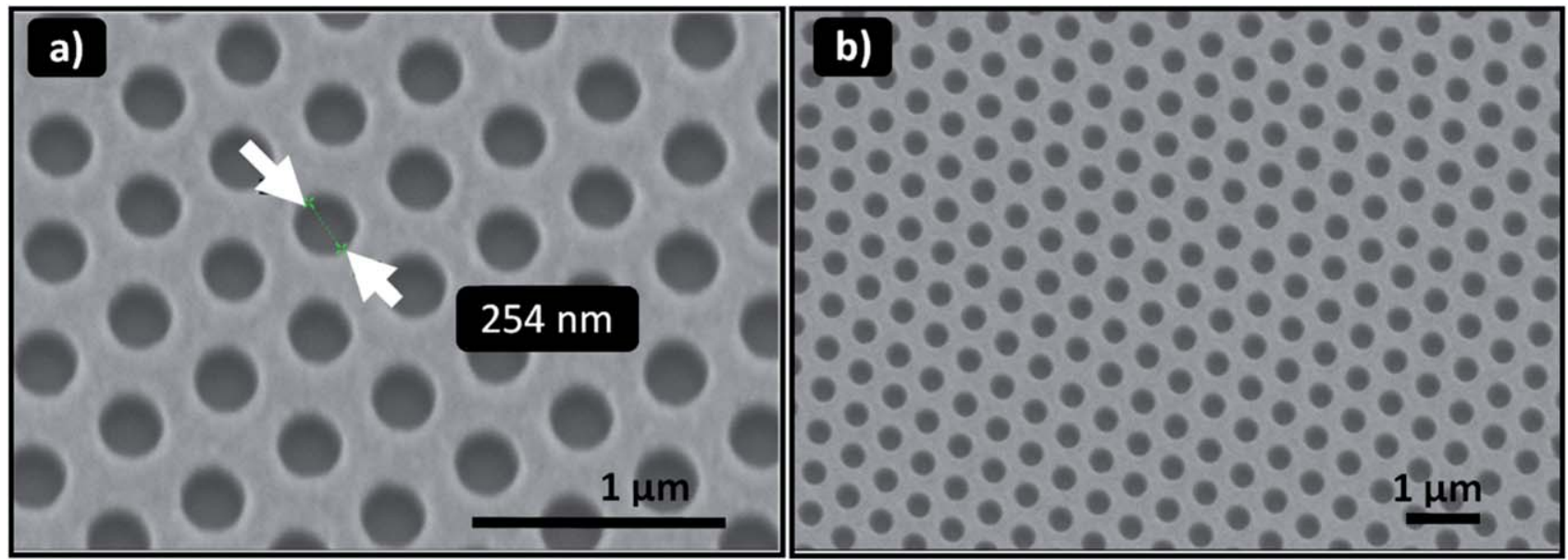

Fig. 7 SEM image of a $50 \mathrm{~nm}$ thick Al film with subwavelength holes: (a) the measured hole diameter was approximately $254 \mathrm{~nm}$, (b) the defect free area ranges to hundreds of $\mu \mathrm{m}^{2}$.

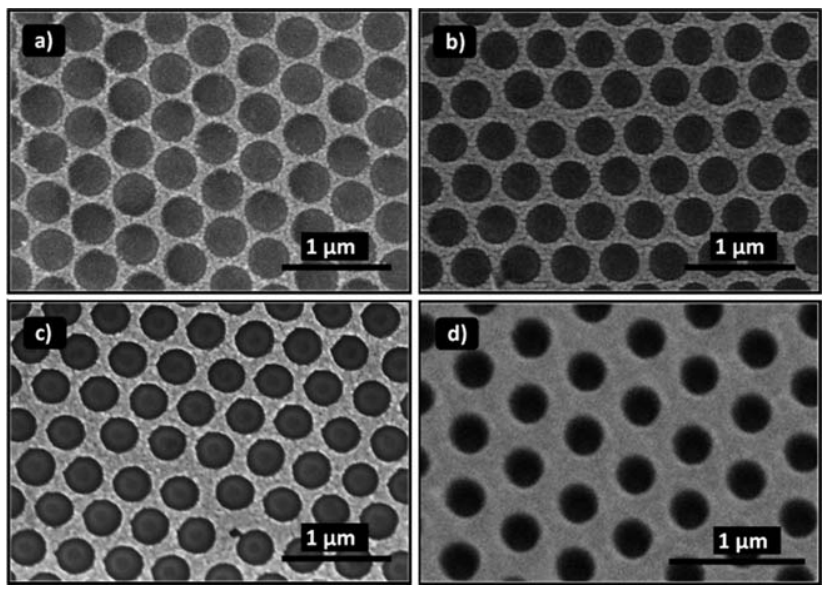

Fig. 8 SEM images of nanohole arrays. The diameter of a single hole is (a) $385 \mathrm{~nm}$, (b) $340 \mathrm{~nm}$, (c) $325 \mathrm{~nm}$ and (d) $254 \mathrm{~nm}$.

polarization. Afterwards the light is coupled into a multimode fiber with a tapered Al-coated tip, the aperture diameter of a fiber tip is between 50 and $100 \mathrm{~nm}$. This offers the opportunity of local illumination of the sample. While working in transmission mode, the light is collected behind the sample through a microscope, and after passing a filter, it is directed to an avalanche photo diode.

\subsection{Gold nanoislands}

In general nanoparticles with nonplatonic shapes (tubes, flakes, rods, prisms etc.) fabricated from different materials and by various techniques have recently attracted a lot of attention ${ }^{16-24}$ mostly because of their interesting optical properties and the large variety of possible applications, i.e., for biosensing, ${ }^{25,26}$ catalysis ${ }^{27}$ or data storage. ${ }^{28}$ Materials that exhibit a large negative real and small positive imaginary dielectric function are capable of supporting a collective excitation of the conduction electrons known as plasmon excitation. In metal nanoparticles this leads to a localized surface plasmon resonance (LSPR). ${ }^{29-31}$ Recent works demonstrated that the position of the LSPR

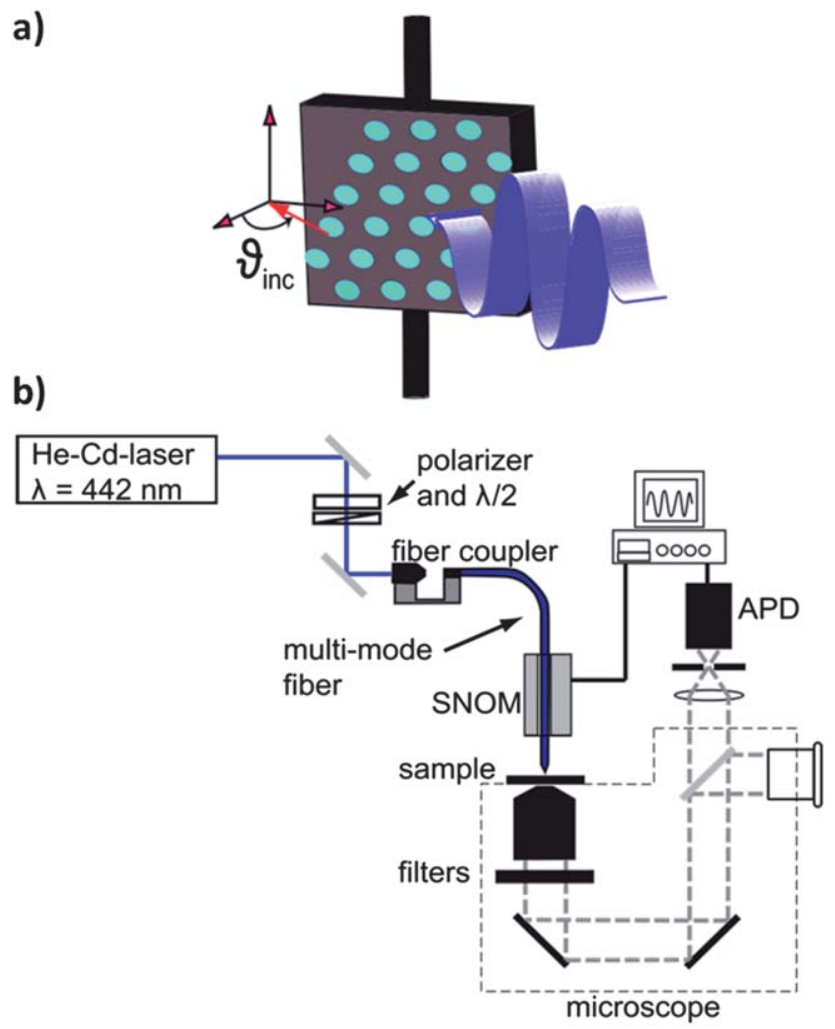

Fig. 9 Schematic of the experimental setup for the optical characterizations: (a) home built sample holder used for far field measurements that enable the change of the incidence angle $\theta$ inc, (b) Schematic of the illumination-mode SNOM measurements in transmission.

extinction maximum, $\lambda_{\max }$, is sensitive to the size, shape, interparticle spacing, dielectric environment, and dielectric properties of the nanoparticles. ${ }^{32-36}$ As a result, metallic nanoparticles that support LSPR are promising platforms as highly sensitive optical nanosensors, as photonic components, and in surface-enhanced spectroscopies. $^{32,37-40}$ It is well established that $\mathrm{Ag}$ and $\mathrm{Au}$ nanoparticles support localized surface plasmon resonances that can be widely tuned from UV to near-IR regions of the 
electromagnetic spectrum..$^{41,42}$ In this section, the plasmonic properties of triangular shaped nanoparticles of different sizes established in a hexagonal array will be investigated.

To obtain a different size of triangular nanoparticles, PMMA (poly-methylmethacrylate) and polystyrene nanospheres with diameters of 380 (PMMA), 540, 980, and $1710 \mathrm{~nm}$ were used. After transferring the mask to sapphire substrates a deposition of $2 \mathrm{~nm}$ Ti (adhesion layer) followed by $20 \mathrm{~nm}$ thick Au films was carried out in an e-beam evaporation-system with a base pressure of $10^{-7} \mathrm{mbar}$. After the cleaning procedure the samples were characterized for structural and optical properties.

The experiments were carried out in the wavelength range between 200-3000 nm under normal incidence of light (no tilting was employed). The experimental spectra for $20 \mathrm{~nm}$ thick $\mathrm{Au}$ triangles on sapphire substrates deposited through masks with sphere diameters $D_{0}=380,540,980$ and $1710 \mathrm{~nm}$ are shown in Fig. 10 (the data were reproduced after ref. 43).

The AFM images on the top of the chart represent the fabricated structure for each $D_{0}$. The aspect ratio between figures is kept, and reflects the difference in structure size. Each spectrum is strongly dominated by a pronounced resonance peak at a wavelength of $704 \mathrm{~nm}, 996 \mathrm{~nm}, 1417 \mathrm{~nm}$ and $2417 \mathrm{~nm}$ respectively (denoted in Fig. 10 for each series with a colored arrow with index 1). The position of each absorption feature strongly depends on $D_{0}$. It is expected, that these resonances are from a highly dispersive, plasmon-polaritonic mode, since similar resonances have been observed in metallic clusters. ${ }^{44,45}$ As it was shown by Peng et al. ${ }^{46}$ one has to assume that these resonances (as well as higher order resonances marked on the chart with an arrow and index corresponding to the order of the

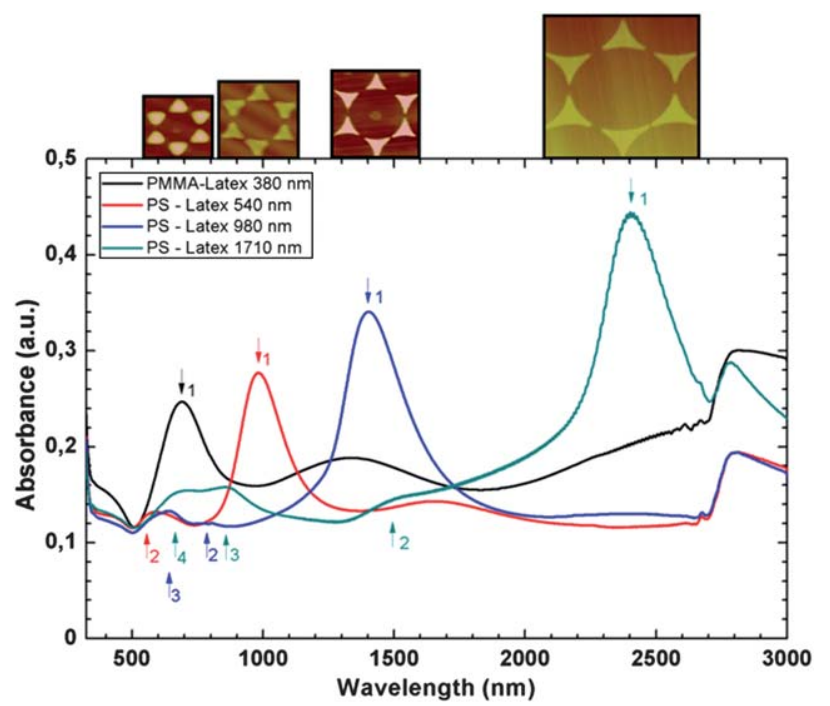

Fig. 10 Measured optical absorbance (in arbitrary units) for the quasitriangle arrays with various $\mathrm{D}_{0}$. The AFM images on the top of the chart represent fabricated structures for each $\mathrm{D}_{0}$. Spectra of the light exhibit first (most pronounced), second, third and forth absorbance peaks for variously sized $\mathrm{Au}$ quasi-triangular particles which were made of different sphere diameter for NSL as presented in a legend of the chart. Arrows with respective index mark absorbance peaks $\left(\lambda_{\max }\right)$ for adequate plasmon resonance order of the triangular particles. The wavelength at the maximum on each curve increases with the sphere diameter $D_{0}=380$, 540, 980, and $1710 \mathrm{~nm}$. resonance) can be viewed as circumferentially quantized surface plasmon waves. We ignore for the moment particle-particle interactions. Since each side of the quasi-triangular particle equals approximately $1 / 6$ of the sphere circumference $\pi D_{0}$, the circumference of the particle is $\pi D_{0} / 2$, and thus the circumferential quantization condition requires that this circumference is equal an integer multiple of the wavelengths of the resonating surface/edge plasmon wave $\left(\lambda_{\mathrm{SP}}\right)$ :

$$
l \lambda_{S P} \approx \pi D_{0} / 2
$$

where $l=1,2,3,4$ is the integer for first (main peak), second, third and forth order of resonance respectively. The other possible interpretations of the peaks at the positions marked as 2nd, 3rd and 4th order for the triangles fabricated with 540, 980, and 1710 $\mathrm{nm}$ respectively is that they belong to the transversal plasmon resonance, which occurs in a thickness of the structure. For the triangles made using $380 \mathrm{~nm}$ spheres this peak is not visible, this could be due to the fact that the main absorbance peak is very pronounced and close enough to overlap small transversal resonance.

In Table 1 the measured $\left(\lambda_{\max }\right)$ and calculated $\lambda_{\text {SP }}$ peak positions for different orders $(l)$ and size of spheres used to fabricate arrays of gold nanoislands are gathered. The values for $\lambda_{S P}$, were obtained by using eqn (1) and the values for $\lambda_{\max }$ were taken from Fig. 10, where wavelengths were read for each arrow-mark (wavelength at a peak) for adequate $D_{0}$.

Fig. 11 shows the resonating mode dispersion plotted as $\omega n /$ $2 \pi c=1 / \lambda_{\text {max }}$ versus $k / 2 \pi=1 / \lambda_{S P}$ and $l . \mathrm{n}$ represents the effective refractive index of the medium which surrounds the nanoparticles (of order 1 in this case). Depending on $D_{0}$ more or less of higher order peaks are visible, i.e., for $D_{0}=1710 \mathrm{~nm}$ four and for $D_{0}=380 \mathrm{~nm}$ only one order is visible. In Fig. 11 the black squares are indexed with numbers indicating the resonance order (for $l=1,2,3,4)$. All of the points congregate around a single line (magenta line in Fig. 11), which is drawn here just as a guide to the eye. This confirms that all of the resonances (measured and calculated) belong to a single mode. The solid blue line represents the corresponding light line (photon dispersion):

$$
\omega=k c / n \text { or simply } 1 / \lambda_{\max }=1 / \lambda_{S P}
$$

For comparison with the experiment, Peng et al.,${ }^{46}$ performed simulations, based on the finite difference time domain (FDTD) computation scheme, and the results are presented in Fig. 11a. Here the white squares represent the simulated dispersion for arrays of triangular-shaped particles. Additionally, a simulated optical absorbance for quasi-triangular arrays is depicted as the

\begin{tabular}{|c|c|c|c|c|c|c|c|c|}
\hline \multirow{3}{*}{$l$} & \multicolumn{8}{|c|}{$D_{0}[\mathrm{~nm}]$} \\
\hline & 380 & & 540 & & 980 & & 1710 & \\
\hline & $\lambda_{\max }$ & $\lambda_{\mathrm{SP}}$ & $\lambda_{\max }$ & $\lambda_{\mathrm{SP}}$ & $\lambda_{\max }$ & $\lambda_{\mathrm{SP}}$ & $\lambda_{\max }$ & $\lambda_{\mathrm{SP}}$ \\
\hline 1 & 704 & 597 & 996 & 848 & 1417 & 1539 & 2417 & 2686 \\
\hline 2 & - & 298 & 596 & 424 & 799 & 769 & 1551 & 1343 \\
\hline 3 & - & 198 & - & 282 & 658 & 513 & 875 & 895 \\
\hline 4 & - & 149 & - & 212 & - & 385 & 709 & 671 \\
\hline
\end{tabular}

\section{Table 1}




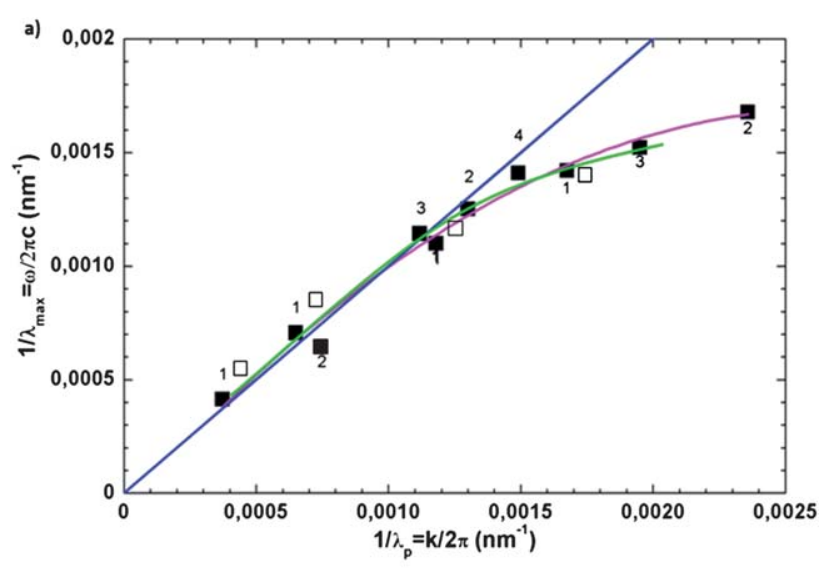

b)

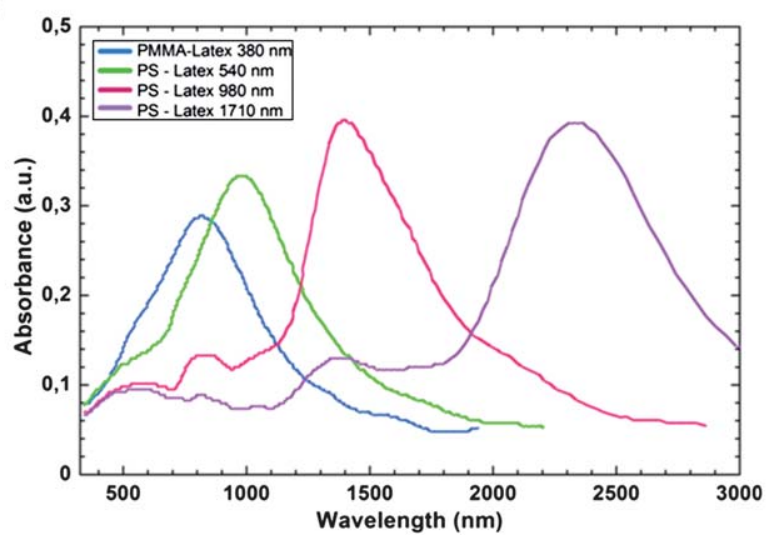

Fig. 11 (a) Measured and simulated dispersion of the resonating surface/edge plasmon in an array of quasi-triangle nanoparticles: measured for all orders, where the numbers by the black squares indicate the corresponding order (for $1=1,2,3,4$ ) respectively. The solid blue line is the light line. The solid magenta line is a fit to the measured data. Simulated dispersion for arrays of triangles is depicted with white squares. The solid green line is a fit to the simulated data. (b) simulated optical absorbance (in arbitrary units) for the quasitriangle arrays with various $\mathrm{D}_{0}$, to be compared to the corresponding experimental data in Fig. 10.

Fig. 11b. The $D_{0}$ chosen for the simulations reflects the sphere diameter used for the experiment. Clearly, all resonances belong to the single mode. The surface/edge plasmon mode in this quasitriangular nanoparticle lattice follows the photon line for wavevectors $\mathrm{k}<0.007 \mathrm{~nm}^{-1}$. This is the retarded, or polaritonic regime, in which the mode is a photon-plasmon hybrid (plasmon polariton). ${ }^{47}$ For large wavevectors $\mathrm{k}>0.007 \mathrm{~nm}^{-1}$ (non-retarded regime), the dispersion curve deviates from the light line. This is due to the presence of the Mie resonance in a center of mass [ref. 46 and references therein], which is the collective motion of the oscillating electron gas in a potential of the positive background. The above mentioned experimental results stand in very good agreement with theoretical calculations as presented in Fig. 11. The fit to the simulated dispersion of the arrays of triangles is nearly identical with the guideline of the measured dispersion. ${ }^{47}$ The discussions above regarding plasmonic features include only features which appear in Fig. 10 for wavelengths equal to and shorter than the main absorbance peak position (different orders of surface/edge plasmon mode). Besides this, there is also an additional feature that can be seen. It is a red-shift and broadening of the resonance according to the 1st order surface/edge plasmon. This peak is noticeable for arrays of triangular particles fabricated with spheres of $D_{0}=380,540$ and $980 \mathrm{~nm}$, at $1345 \mathrm{~nm}$, $1675 \mathrm{~nm}$, and $2414 \mathrm{~nm}$, respectively. For the spheres of $D_{0}=$ $1710 \mathrm{~nm}$ this peak is not present because it is a secondary maximum beyond detector range. These features are most likely an outcome of connected triangles which come from imperfections which are direct results of the quality of self-organized latex particles. Following measurements of the triangular shaped nanoislands a characterization of the perforated films has been carried out.

\subsection{Gold and aluminum subwavelength holes}

The transmission properties of a subwavelength hole in a metal film have been studied for decades. However, the most intensive investigations have been carried out after Ebbesen and coworkers experimentally showed that the extension of a single hole to an array of subwavelength apertures leads to an enhanced transmission of light. Since the first predictions for a single aperture published by Bethe in $1944,{ }^{48}$ many attempts to understand the transmission properties which are fundamental in near-field optical microscopy were carried out. In addition to this, a huge amount of theoretical ${ }^{49-51}$ and experimental ${ }^{52-55}$ studies have been performed to understand the underlying physics. The results of the transmission measurements on these subwavelength hole arrays draw the attention to nanostructure films. There was a need to find the origins of the processes responsible for the reported enhanced transmission. There is still a lot of discussion and disagreement on the origin of the underlying physics describing the extraordinary transmission. The two approaches are: the model of coupling of light to surface plasmons ${ }^{56-58}$ and the model of evanescent waves. ${ }^{59}$ Finally, it was clarified ${ }^{60}$ with advantage to the model of coupling to SP, which is connected to the geometrical parameters of the array of holes. Thus the change of parameters like hole depth and period has an influence to the plasmonic response. Still the recent works report only on results of optical properties of arrays with square symmetry of holes in films.

Therefore, in the following sections the experimental results on hexagonal arrays of holes in a gold and aluminum film will be presented. The characterization was carried out on hole arrays in gold and aluminum films of thickness 20, 50 and, $120 \mathrm{~nm}$. For the $\mathrm{Au}$ films a buffer layer of $2 \mathrm{~nm}$ Ti was used, as in the case of nanotriangles. The measured hole diameter $D_{H}$ is $250 \mathrm{~nm}$ and the interhole distance is kept constant at $470 \mathrm{~nm}$.

4.2.1 Far field results. The far-field optical characterization results measured in the range between 200 and $2500 \mathrm{~nm}$ are shown in Fig. 12. The measurements show transmission spectra of samples taken under normal incidence, for a gold (panel a) and an aluminum (panel b) nanostructured film. Additionally, the spectra of $20 \mathrm{~nm}$ thick film (blue lines) are included for comparison. As expected, the solid and structured gold films show a pronounced peak at $2.5 \mathrm{eV}(\sim 500 \mathrm{~nm})$ as a spectral feature whose origin is quite complex. Gold material as well as copper exhibit considerable overlapping of surface plasmon resonance and interband transition in the visible range. ${ }^{61}$ Here 

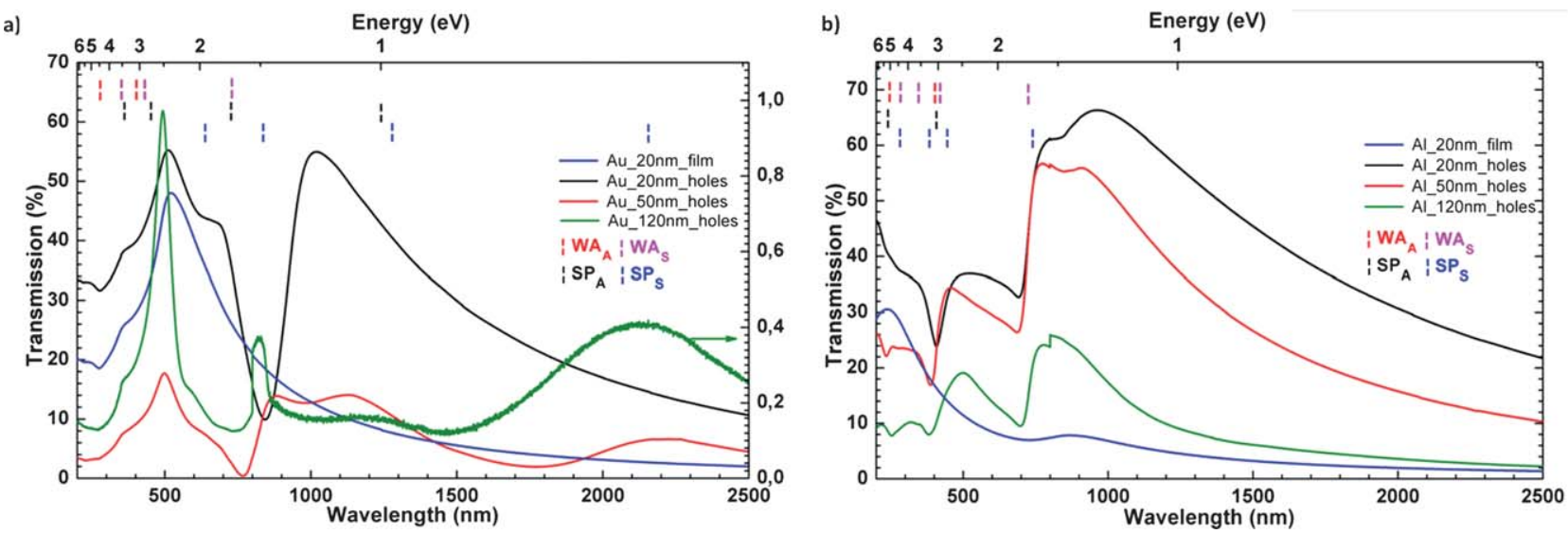

Fig. 12 UV-Vis-NIR transmission spectra: (a) gold and (b) aluminum films with a thickness of $20 \mathrm{~nm}$ (blue line) and films with hole arrays showing differences in their spectral features. The film thickness is $20 \mathrm{~nm}, 50 \mathrm{~nm}$ and $120 \mathrm{~nm}$ and is represented with black, red, and green color line respectively. The red, pink, black and blue dashed lines denote the calculated positions at which Wood's anomaly and surface plasmons are expected. The A and S letters stand for the metal-air and metal-sapphire interfaces respectively.

one can observe the rising edge from $2.3 \mathrm{eV}(540 \mathrm{~nm})$ towards shorter wavelengths which is due to the bulk plasmon edge following with interband transition trough at around $2.5 \mathrm{eV}$, where d-electrons are promoted to the s-p conduction band. ${ }^{62}$ The peak broadens slightly with decreasing film thickness. Such an effect is very pronounced in case of small nanoparticles, when the particle's radius is reduced, the conduction electrons (which are almost free) experience collisions not only with positive background but also with the surface which explains the broadening of the surface plasma resonance peak. Some additional features are visible in the spectra of gold hole arrays at 820 $\mathrm{nm}, 870 \mathrm{~nm}$, and $1000 \mathrm{~nm}$ for all film thicknesses. These peaks tend to red shift and broaden with decreasing film thickness. The width of the peaks appears to be strongly dependent on the aspect ratio, i.e., the film thickness divided by the diameter of the cylindrical holes $\left(\mathrm{t} / D_{H}\right)$. For a film thickness to hole diameter ratio close to 0.1 , the peaks are very broad and only just discernible. When the ratio reaches $\sim 0.5$, the maximum sharpness is obtained. Further narrowing might depend on the quality of the individual holes. ${ }^{56}$ In the study done by Ebbesen, he also claims that an array of such holes can present spectra with high zero-order transmission (where the incident and detected light are collinear) at wavelengths which are longer than the period of the array $(470 \mathrm{~nm})$, beyond which no diffraction can occur. In our case the additional interesting features are the two pronounced peaks above $1000 \mathrm{~nm}$ which in fact is much longer than the hole diameter: the first one is centered around $1200 \mathrm{~nm}$ and the second one around $2100 \mathrm{~nm}$, respectively. One can observe that while the film thickness is decreasing, the peak intensity increases with the peak at $1100 \mathrm{~nm}$ and decreases with the peak at $2100 \mathrm{~nm}$. Finally, for the $20 \mathrm{~nm}$ gold film only the first, strongly pronounced peak at $1000 \mathrm{~nm}$ remains. This behavior points towards a change in the oscillator strength.

Other additional features for arrays of holes can be connected to surface plasmons (SP) at maxima closely followed by Wood anomalies at minima positions. ${ }^{55,57,63}$ To prove this statement, we have calculated wavelengths with eqn (3) (where $a_{0}$ is the periodicity of the array, $\varepsilon_{m}$ and $\varepsilon_{d}$ are the real parts of the dielectric constants of the metal and the dielectric, the $i$ and $j$ are integers denoting the order of the SP resonances at which the surface plasmons are expected for both interfaces and are depicted with blue and black dashed lines in Fig. 13a.

$$
\lambda \sqrt{\frac{4}{3}\left(i^{2}+j^{2}+i j\right)}=a_{0} \sqrt{\frac{\varepsilon_{m} \varepsilon_{d}}{\varepsilon_{m}+\varepsilon_{d}}}
$$

with $a_{0}$ is the periodicity of the array, $\varepsilon_{m}$ and $\varepsilon_{d}$ are the real parts of the dielectric constants of the metal and the dielectric, the $i$ and $j$ are integers denoting the order of the SP resonances. The peak positions for the metal-air interface are labeled as $\mathrm{SP}_{\mathrm{A}}$. The label $\mathrm{SP}_{\mathrm{S}}$ is related to the peak positions for the metal-substrate interface. Additionally, for both dielectric materials boundaring with the hole arrays, the positions of Wood's anomaly are calculated with eqn (4) and depicted in Fig. 12a.

$$
\lambda_{W A}=\frac{a_{0}}{\sqrt{\frac{4}{3}\left(i^{2}+j^{2}+i j\right)}} \sqrt{\varepsilon_{d}}
$$

Here, red dashed lines labeled as $\mathrm{WA}_{\mathrm{A}}$ and magenta dashed lines labeled as $\mathrm{WA}_{\mathrm{S}}$ represent positions for air and substrate respectively. One has to keep in mind that eqn (3) does not take into account the physical presence of the holes; it only makes use of an array. It also does not consider the associated scattering losses. Besides, it neglects the interference between a direct transmission of light through the holes predicted by Bethe and the resonant transmission due to the excitation of surface plasmons, which can manifest with presence of Fano profiles on a spectra ${ }^{64}$ and a red shift of the resonance. ${ }^{65}$ The consequence of omission of these facts is that calculated peak positions are at wavelengths slightly shorter than in experimental data presented in Fig. 12.

For the first approximation our calculations explain the features for wavelengths longer than $500 \mathrm{~nm}$ in the case of the 50 $\mathrm{nm}$ and $120 \mathrm{~nm}$ thick gold hole arrays [Fig. 12a, red and green line]. As it was mentioned before, at around $500 \mathrm{~nm}(2.5 \mathrm{eV})$, which is the wavelength region of the surface plasmon and interband transition, the transmission is totally intermixed with peaks related to Wood's anomaly forming a complex pattern. 

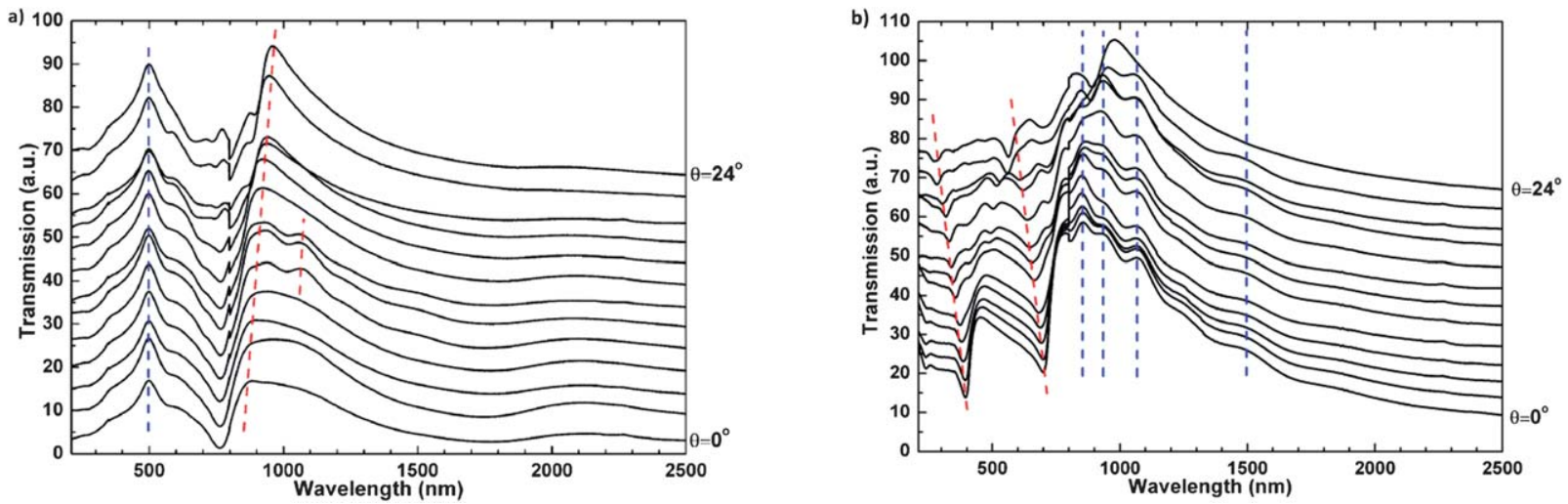

Fig. 13 Angle of incidence dependent optical spectra of a $50 \mathrm{~nm}$ thick perforated film are shown. Blue and red dashed lines mark the features of spectra remaining and changing their positions for: (a) gold and (b) aluminum as film material, respectively.

Since this very simple single-interface-at-a-time approach is justified only for films that are much thicker than the field penetration depth $(\sim 20 \mathrm{~nm})$, some discrepancy is expected for the $20 \mathrm{~nm}$ film. For this optically very thin film, a strong contribution resulting from the direct transmission is expected. The shift of the peak is attributed to the coupling of the surface plasmons on both interfaces of the film and thus directly to the thickness of the metal film. ${ }^{66,67}$

Fig. 12b shows the spectra for aluminum films. Once again the blue line represents the transmission spectrum for a solid film with a thickness of $20 \mathrm{~nm}$. Here at $800 \mathrm{~nm}(1.5 \mathrm{eV})$ the known peak related to interband transition is pronounced. ${ }^{68,69} \mathrm{In}$ contrast to the gold spectra, the spectra for the aluminum films showed essentially similar results for the different film thicknesses, except for the overall scaling. Additionally, there are no pronounced peaks related to a surface plasmon overlapped with interband transition edge in the visible range. Furthermore, a bulk plasmon for Aluminum appears at high energy $\left(15.2 \mathrm{eV}^{70}\right.$ which lies beyond our interest). Unlike in the case of gold we did not observe any features in the strongly subwavelength regime, i.e., for wavelengths larger than $1000 \mathrm{~nm}$. The features below $750 \mathrm{~nm}$ are most likely due to Wood's anomalies. Both troughs near 700 and $400 \mathrm{~nm}$ have a Fano profile, pointing towards Wood anomalies mediated by surface plasmons. As for gold we have performed calculations to theoretically find the wavelengths at which the surface plasmons as well as Wood's anomaly could be present.

The calculated values are plotted on Fig. 12b with the same symbols and color-scheme as for the gold samples. One can see that theoretical calculations stand in perfect agreement with experimental results (keeping in mind the slight red shift whose origin has been discussed before). It has been revealed that the feature at $750 \mathrm{~nm}$ is not just the result of broad interband transition peak. Here, we encounter the same situation as for the gold perforated films; the same situation as in the case of Au perforated films. The interband transition raised a peak attributed to a surface plasmon resonance followed by a trough at $724 \mathrm{~nm}$ being a direct result of Wood's anomaly forming a Fano profile together. The same explanation applies to features which are present at higher energies. Here the most emphasized is a peak at around $500 \mathrm{~nm}$ and a trough at around $400 \mathrm{~nm}$. In overall scaling, one can observe that all of the features tend to shift towards shorter wavelengths while the perforated film thickness is increased. This is the effect of the aspect ratio between the holes diameter and their depth, which is the same as described for the gold samples.

Additionally for both materials angle-dependent transmission measurements have been performed for the $50 \mathrm{~nm}$ thick films (Fig. 13). Even small changes in the incidence angle marked changes in zero-order transmission spectra which were recorded every $2^{\circ}$. In the case of $\mathrm{Au}$, at around $1000 \mathrm{~nm}$, a change in intensity and a shift of the structure with simultaneous peak splitting and movement in opposite directions is recognizable (Fig. 13 a red dotted line). Such behavior can indicate the development of a band structure.

To confirm this statement, angle dependent measurements were performed, mapping the optical band structure for incident angles up to $24^{\circ}$ along the $\Gamma \mathrm{K}$-direction of the hexagonal array [Fig. 14]. In the graph, the evolution of the peak at around $1000 \mathrm{~nm}$ can be followed. Additionally, the calculated dispersion

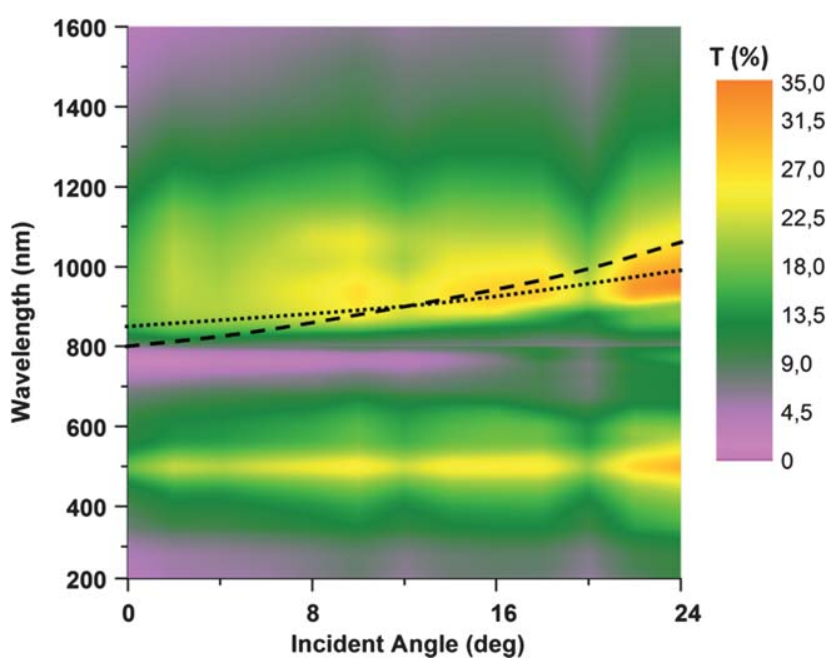

Fig. 14 Angle dependent measurements of $50 \mathrm{~nm}$ thick gold perforated film along the $\Gamma$ K-direction of the hexagonal array. The dotted line denotes the evolution of the measured SP peak, dashed line-theoretically calculated dispersion of the SP $(2,1)$ mode for the metal-substrate interface. 
relation for the SP 2-1 mode (for the metal-substrate interface extended for the different incidence angles ${ }^{71}$ eqn (3)) is included in the picture (dashed line). This calculated curve matches the measured (dotted line) dispersion very well and indicates the creation of the band structure; more precisely a plasmonic band structure since this peak is attributed to a surface plasmon. The peak at $500 \mathrm{~nm}$ remains on its position while the incidence angle is changed (blue dotted line in Fig. 13a). For a $50 \mathrm{~nm}$ thick perforated $\mathrm{Al}$ film, the two high-energy troughs at 400 and 700 $\mathrm{nm}$ respectively are shifting (Fig. 13b red dotted lines) with increasing $k$ parallel $\left(k_{x}=\left|\vec{k}_{0}\right| \sin \theta\right)$. To prove the correct interpretation of these features, the results of angle dependent measurements were presented as a map of optical response, where one can follow the evolution of the recorded peaks and troughs [Fig. 15]. The changing positions of the surface plasmons are marked with black dashed lines indexed with the corresponding mode number. Wood's anomaly related troughs are denoted with black dotted lines. Due to the change of the angle of incidence one can observe that Wood's anomaly gives rise to a set of minima which closely follow the measured maxima of the SP dispersion. The recorded surface plasmon dispersion curves for the aluminum film with subwavelength holes indicate the creation of the plasmonic band structure, as it was presented in the case of gold samples. For a more detailed study of the origin of enhanced transmission, near-field measurements were performed. The results of these measurements are presented in the following section.

4.2.2 Near field results. Following the far-field characterization of the $\mathrm{Au}$ and $\mathrm{Al}$ hole arrays, we have carried out studies on the near-field optical behavior of the light transmission to determine and point out the main transmission channel for the light through the perforated film. The experiments were performed in illumination mode, giving the opportunity to locally

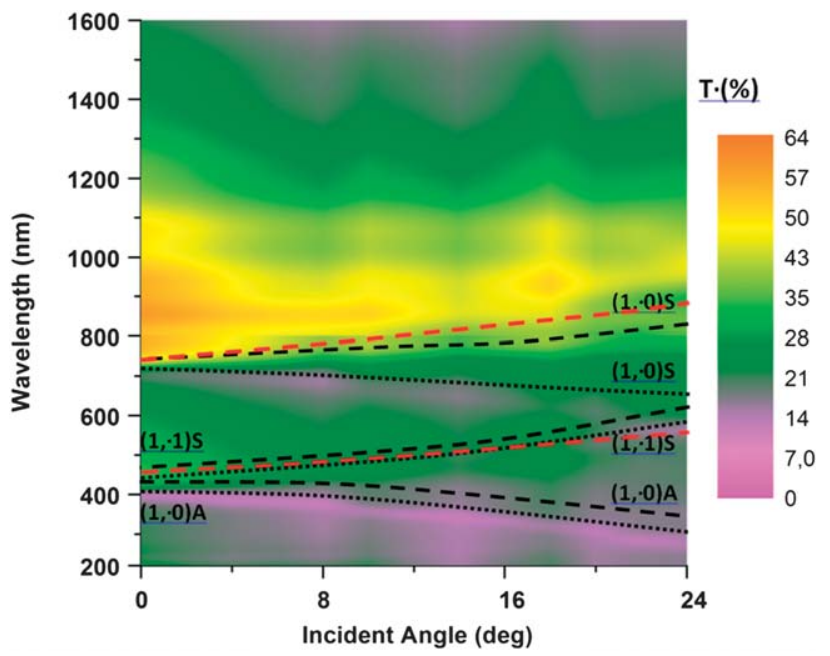

Fig. 15 Angle dependent measurements mapping of $50 \mathrm{~nm}$ thick aluminum perforated film. The black dashed lines denote the evolution of the measured surface plasmons peaks and dotted lines indicate the Wood's anomaly troughs, excited on: S-metal-substrate and A-air-metal interface. The red dashed lines represent the calculated dispersion of $(1,0)$ $\mathrm{S}$ and $(1,1) \mathrm{S}$ surface plasmons.
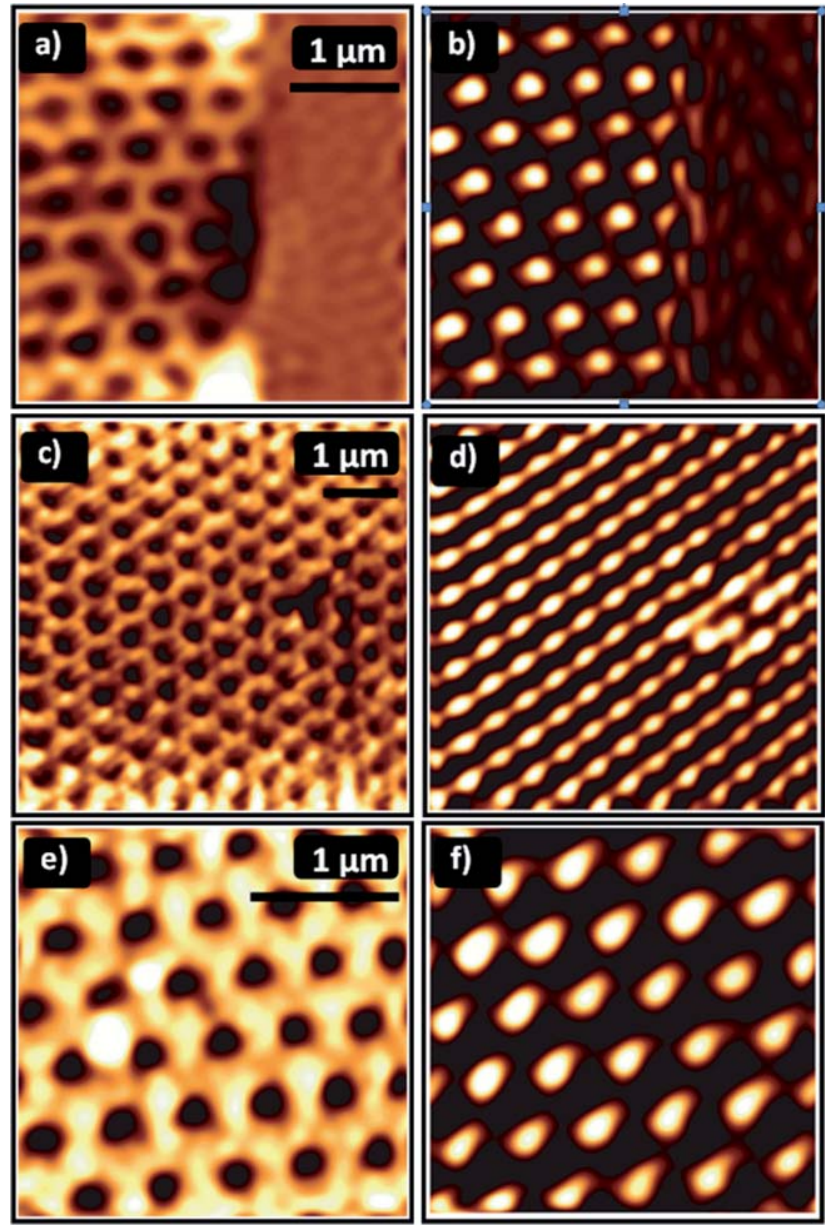

Fig. 16 SNOM images of the Au hole arrays. Left column: Topography images of the samples indicating an interhole distance of $\sim 500 \mathrm{~nm}$ and a hole diameter of $250 \mathrm{~nm}$. Right column Near-field optical images taken at a wavelength of $442 \mathrm{~nm}$. The recorded light intensity shows an enhanced transmission through the sub-wavelength holes. The thickness of the deposited films is $20 \mathrm{~nm}(\mathrm{a}, \mathrm{b}), 50 \mathrm{~nm}$ (c, d), and $120 \mathrm{~nm}(\mathrm{e}, \mathrm{f})$, respectively.

illuminate the sample using a wavelength of $442 \mathrm{~nm}$. In Fig. 16, the results for the gold nanohole arrays with a different film thicknesses of -20 [(a) and (b)], 50 [(c) and (d)], and $120 \mathrm{~nm}$ [(e) and (f)], are presented. The hole diameter of all samples was set to $250 \mathrm{~nm}$. The topography images (left column) were taken simultaneously with optical images (right column). Comparing now the simultaneously recorded optical and topography images reveals a high transmission of the light directly at the position of the holes. This effect is observable for all of the film thicknesses and might be explained by the direct light transmission through these subwavelength holes, which is enhanced and supported by the excitation of surface plasmons. The optical images also reveal an elongation of the circular hole-shaped light spots especially in the case of the two thinner films, which on first look would point to a tip-artifact. However, this is a direct result of the polarization of the incoming light. The elongation is parallel to the direction of the polarization and indicates coupling of the locally launched surface plasmons by the tip of a scanning near-field optical microscope ${ }^{72,73}$ and SPs at the film-air interface. These surface plasmons propagate along the surface of the film until 
they encounter a hole. There they are scattered into a radiative mode, possibly by direct interaction with the edges of the holes. For the thinner films, i.e., 20 and $50 \mathrm{~nm}$ thickness, the more probable scenario is that they directly couple to SP modes on the opposite interface of the film and are then scattered into radiative modes. ${ }^{74}$

In contrast to the surface plasmon contribution to the measured light intensity, the intensity of the central spots in Fig. 16b, $\mathrm{d}$, and $\mathrm{f}$ is essentially independent of the light polarization. Thus this peak is attributed to direct transmission through the hole of propagating light modes emitted by the SNOM tip. Following the thorough studies on gold structured films, near-field optical transmission through apertures in aluminum films was investigated for comparison. The results are presented on Fig. 17; identical as in the case of gold samples a left column represents topography images for $20 \mathrm{~nm}$ [(a)], $50 \mathrm{~nm}$ [(c)] and $120 \mathrm{~nm}[(\mathrm{e})]$ thick aluminum films. The right column reflects optical signal measurement results for $20 \mathrm{~nm}$ [(b)], $50 \mathrm{~nm}$ [(d)], and $120 \mathrm{~nm}$ [(f)] which are adequate thicknesses taken
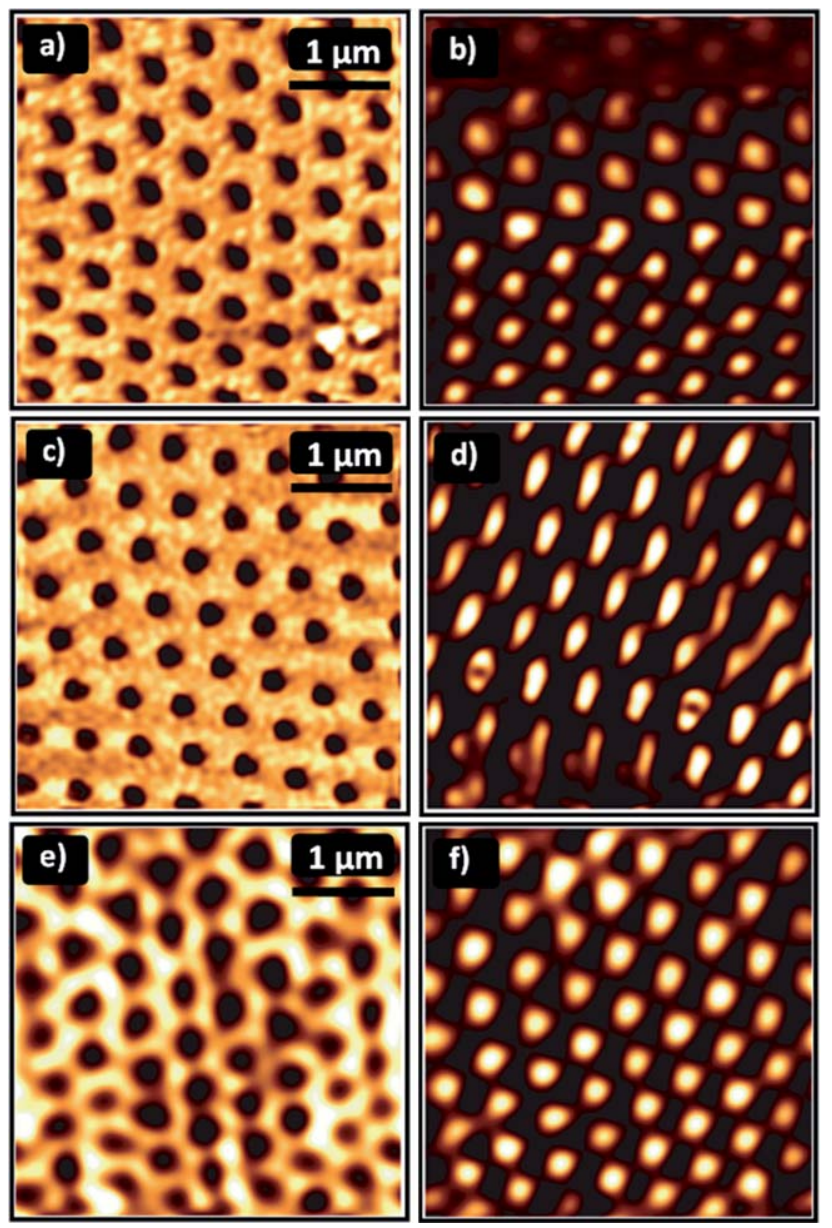

Fig. 17 SNOM images of the hole arrays in the Al films. Left column: topography images of the samples indicating an inter-hole distance of $\sim 500 \mathrm{~nm}$ and a hole diameter of $250 \mathrm{~nm}$. Right column: Near-field optical images at a wavelength of $442 \mathrm{~nm}$. The recorded light intensity again shows an enhanced transmission through the sub-wavelength holes. The thicknesses of the deposited films are $20 \mathrm{~nm}(\mathrm{a}, \mathrm{b}), 50 \mathrm{~nm}(\mathrm{c}, \mathrm{d})$, and $120 \mathrm{~nm}(\mathrm{e}, \mathrm{f})$, respectively. simultaneously with topography, thus giving the films the same thicknesses as the gold equivalents. Exactly as in the case of gold, the observed contrast shows an enhanced transmission of light through the holes and is mostly the result of a direct transmission through the hole of propagating light, with surface plasmon enhancement. In Fig. 17, similar to Fig. 16 one can observe the elongation of the spots of transmitted light at the position of the holes. This is again connected to the polarization direction. In addition, the influence of the present native oxide layer on those aluminum films was not taken into account, leaving room for further experiments and theoretical calculations to clarify its possible role in the transmission enhancement.

To better understand the processes governing the light propagation through our hole arrays, 3D FDTD simulations ${ }^{75}$ were performed in the group of Prof. Kempa (Boston College), modeling the field distribution in our films. ${ }^{76}$ They have used periodic boundary conditions associated with the hexagonal hole structure, and absorption conditions to truncate the directions parallel to the film surface. The thickness of the film was taken to be $50 \mathrm{~nm}$, the inter-hole distance is $500 \mathrm{~nm}$, and the diameter of the holes $d=250 \mathrm{~nm}$, to match the experimental conditions. The discretization step size of $2 \mathrm{~nm}$ assures good numerical convergence. The time-domain auxiliary differential equation approach was used to implement the FDTD method to our case of the dispersive metal. The frequency-dependent permittivity of $\mathrm{Au}$ was taken from ref. 74. The in-plane field intensity profiles shown in Fig. 18 are calculated in a single unit cell, at $10 \mathrm{~nm}$ away from

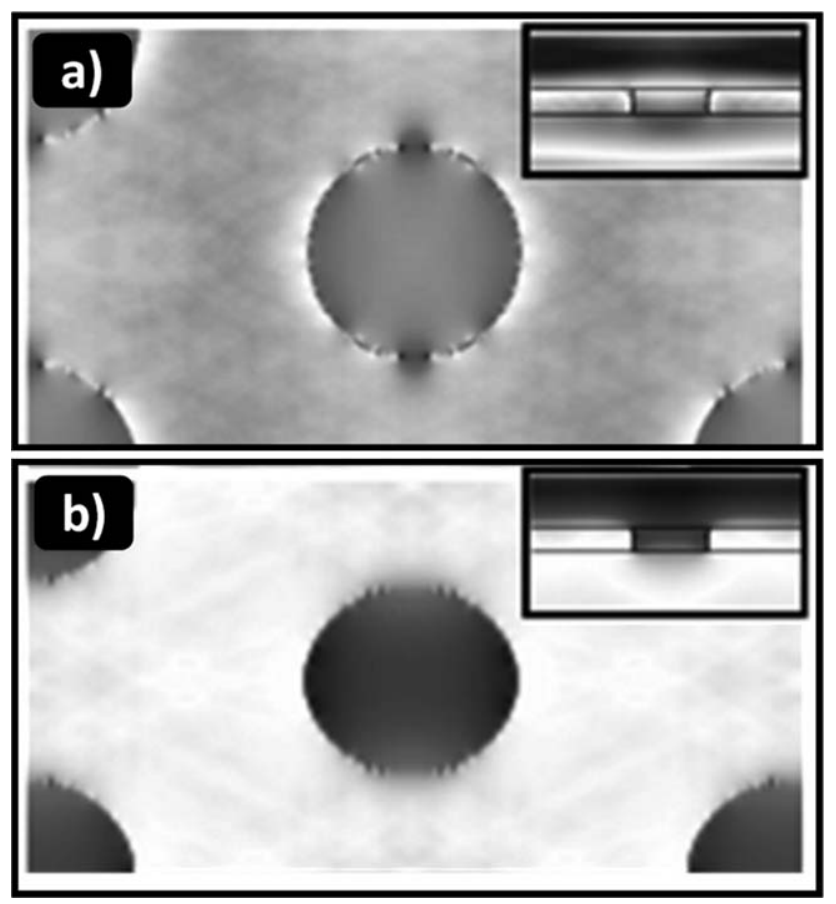

Fig. 18 Simulation of the light transmission through the Au film with an array of holes. Parameters: inter-hole distance: $500 \mathrm{~nm}$, hole diameter $250 \mathrm{~nm}$, film thickness $50 \mathrm{~nm}$, wavelength of the light is $442 \mathrm{~nm}$ (a), and $1000 \mathrm{~nm}$ (b). Main figures show light intensity $10 \mathrm{~nm}$ away from the film surface on the far side. Darker regions represent higher intensity. Insets show the corresponding in-plane electric field amplitudes (taken from ref. 76). 
the film surface, on the far side of the film. The chosen colorcoding scheme shows high-intensity regions in a darker color. The light wavelength is $\lambda=442 \mathrm{~nm}$ in Fig. 18a, and $\lambda=1000 \mathrm{~nm}$ in Fig. 18b. The insets show the corresponding in-plane electric field amplitude profiles across the film. In the marginal subwavelength regime $(d<\lambda$, Fig. 18a), the overall transmission of light through the film and holes is very high (mostly due to the plasmon enhancement), as shown in the inset. The corresponding in-plane intensity map shows that the transmission is larger inside the holes, which stays in a very good agreement with the experiment. In the strongly subwavelength regime $(d \ll \lambda$, Fig. 18b), the light propagation through the metal is marginal (in part since little plasmon enhancement occurs in this range), but is quite large through the holes. This is obvious from the inset showing out of plane field intensity, as well as, the corresponding in-plane intensity map. An overall transmission coefficient through this film at this wavelength $(\lambda=1000 \mathrm{~nm})$ is much smaller than that for $\lambda=442 \mathrm{~nm}$, as evidenced by the insets in Fig. 18a and b. A maximum in transmission coefficient is indeed observed at $\lambda=442 \mathrm{~nm}$ in all curves in Fig. 12a, followed by a smaller, much broader maximum at $\lambda=1000 \mathrm{~nm}$.

\section{Conclusions}

The present work introduces shadow nanosphere lithography as a time-efficient and low-cost lithographic method to produce large arrays of nanostructures. We thereby use 2D colloidal crystals made of polystyrene (PS) spheres that serve as the lithographic mask. Using different materials and evaporating them at different angles onto the mask leaves the freedom of producing nanostructures of various shapes, for which investigations of optical properties were carried out. It has been presented that structures of different morphologies and made of different materials exhibit interesting optical response on the incident light. It appears that these responses are mainly driven by the excitation of the surface plasmons.

We have presented far field characterization of arrays of triangular shaped nanostructures made by deposition of gold material through masks assembled by use of different sizes of spheres. Such a structure exhibits a strong localized surface plasmon response, whose spectral position as well as number of excitations is strongly dependent on the circumference of single nanostructure. This geometrical parameter can be well controlled by use of desired sphere size for mask assembling. We thus have a tool enabling the manipulation of the plasmonic response of such a structure. Also presented is the fact that depending on the order of the excited plasmon, these can bring about different effects. Lower orders are surface/edge plasmons and higher orders are related to Mie resonances.

The modification of the colloidal mask by use of reactive ion etching allows us to prepare arrays of subwavelength holes with hexagonal symmetry. The type of symmetry differs from the squared lattices of holes being under a great interest in the last decade because of their enhanced light transmission properties. Our investigations in far-as well as in near-field showed that our mesh, with single hole diameters of $250 \mathrm{~nm}$, which were made of gold and aluminum, also exhibit extraordinary transmission of light. The spectral responses are dominated by two phenomena, namely: surface plasmons and Wood's anomalies, which are strongly dependent on samples' material and geometric parameters. The near-field measurements carried out by scanning near-field optical microscopy revealed the transmission of light through the subwavelength holes. This is possible due to the coupling of surface plasmons excited on both sides of the metal film (air-metal and metal-substrate interface). This statement is confirmed by theoretical simulations. The response can be tuned by proper choice of sample's material and morphology.

\section{Outlook}

The presented optical characteristics of different types of structures which were mainly made of gold are just a small part of measurements that can be done.

All of the experimental results fit very well to the proposed theories on surface plasmon excitation in different types of nanostructures presented in this work. It will be very useful to test these theories for nanostructure arrays of different morphologies and various materials. We have shown that use of magnetic material for creation of the hole array, can influence the optical response of the structure; these results are not presented in this work. The light transmission through such a film strongly depends on the external magnetic field. The change of this field influences and tunes the position of the surface plasmons which govern the transmission of the light. We were one of the first group to report on these magneto-plasmonic experimental measurements. ${ }^{71,77,78}$

Change of some structural parameters, i.e., hole diameter, interhole distance, spacing, periodicity, height (thickness) can have a tremendous influence on the optical response of the samples in far field spectroscopy. Further investigations concerning the wavelength dependence of the transmission in the near field can be a valuable reference in detailed understanding of proceeded effects.

It was presented that structural parameters of the samples can be well controlled with NSL giving an opportunity to prepare not only the nanostructures of the same morphology and different geometrical parameters but also of different shape. Combining it with a material deposition technique such as electron beam evaporation, where a large number of materials can be used, opens up a vast space of possibilities. It will be very interesting to investigate how polycrystallinity of the structures influences the plasmonic response of the samples in comparison to monocrystalline samples, i.e., how scattering losses and random scattering can alter the excitation and propagation of the surface plasmon polaritons. It is known that monocrystalline structures cannot be prepared by electron beam evaporation. Therefore, use of some post processing such as thermal annealing should be applied. Nanosphere lithography allows the preparation of masks on various substrates, thus gives us a direct control on the dielectric environment of the fabricated structures. The change of the $\varepsilon_{\mathrm{d}}$ leads to changes in the surface plasmon position in the spectra as shown (eqn (3)).

It would be also very interesting to separate the direct transmission of light from that assisted by plasmon. This could be achieved by use of grazing incident light. Thus, one should observe the vanishing of the Fano profile of the resonance in the spectrum. 
Subwavelength holes can be used as cavities which can be filled with nanoparticles or quantum dots. This procedure will locally affect the dielectric function. Thereby a change in the transmission efficiency or the plasmonic response of the holes is expected. Furthermore, in the case of quantum dots, one expects a change in the emission rate through the changed local density of states of the surrounding medium.

The variety of future experiments can reveal many interesting properties of such nanostructures, which can be used for application in many scientific as well as industrial areas like: optoelectronics, optical lithography, biotechnology.

\section{Acknowledgements}

PP thanks to Max-Planck Institute for Colloids and Surfaces in Golm for the financial support and to all members of Prof. Michael Giersig's group for their help during PhD studies. MG thanks for financial support from the Nanoscale Project FUBerlin and Helmholtz Zentrum Berlin.

\section{Notes and references}

1 J. Rybczynski, M. Hilgendorff, M. Giersig, Nanosphere Lithography-Fabrication of Various Periodic Magnetic Particle Arrays using Versatile Nanosphere Masks, in: NATO Science Series II, 91,/Low-Dimensional Systems: Theory, Preparation, and Some Applications/, L. M. Liz-Marzán, M. Giersig, ed., Kluwer Academic Publishers, Dordrecht, 2003, p. 163.

2 A. Kosiorek, W. Kandulski, P. Chudzinski, K. Kempa and M. Giersig, Nano Lett., 2004, 4, 1359.

3 A. Kosiorek, W. Kandulski, H. Glaczynska and M. Giersig, Small, 2005, 1(4), 439.

4 H. W. Deckman and J. H. Dunsmuir, Appl. Phys. Lett., 1982, 41, 377. 5 M. Giersig and P. Mulvaney, Langmuir, 1993, 9(12), 3408.

6 J. C. Hulteen and R. P. Van Duyne, J. Vac. Sci. Technol., A, 1995, 13,3 .

7 M. Winzer, M. Kleiber, N. Dix and R. Wiesendanger, Appl. Phys. A, 1996, 63(6), 617.

8 R. Micheletto, H. Fukuda and M. Ohtsu, Langmuir, 1995, 11(9), 3333.

9 F. Burmeister, W. Badowsky, T. Braun, S. Wieprich, J. Boneberg and P. Leiderer, Appl. Surf. Sci., 1999, 144-145, 461.

10 N. D. Denkov, O. D. Velev, P. A. Kralchevsky, I. B. Ivanov, H. Yoshimura and K. Nagayama, Langmuir, 1992, 8(12), 3183.

11 W. Kandulski, Shadow Nanosphere Lithrraphy, PhD Thesis, Rheinischen Friedrich-Wilhelms-Universität Bonn, 2007.

12 W. Kern and D. Puotinen, RCA Review, 1970, 31, 187.

13 M. Sugawara, Plasma Etching: Fundamentals and Applications, Oxford University Press, 1998.

14 M. J. Madou, Fundamentals of microfabrication, the science of miniaturization, second edition, CRC Press, London, 2002.

15 P. Patoka, Tunable plasmonic properties of nanostructures fabricated by shadow nanosphere lithography, $\mathrm{PhD}$ Thesis, Freie Universität Berlin, 2011.

16 A. Sánchez-Iglesias, I. Pastoriza-Santos, J. Pérez-Juste, B. RodríguezGonzález, F. J. García de Abajo and L. M. Liz-Marzán, Adv. Mater., 2006, 18, 2529.

17 C. J. Johnson, E. Dujardin, S. A. Davis, C. J. Murphy and S. Mann, J. Mater. Chem., 2002, 12, 1765.

18 C. H. Chen, J. Wang, H. Yu, H. Yang, S. Xie and J. Li, J. Phys. Chem. $B, 2005, \mathbf{1 0 9}, 2573$.

19 Z. L. Wang, M. B. Mohamed, S. Link and M. A. El-Sayed, Surf. Sci., 1999, 440, L809.

20 I. Kirkland, P. P. Edwards, D. A. Jefferson and D. G. Duff, Annu. Rep. Prog. Chem., Sect. C, 1990, 87, 247.

21 R. Jin, C. Y. Cao, E. Hao, G. Métraux, G. C. Schatz and C. A. Mirkin, Nature, 2003, 425, 487.

22 C. Lofton and W. Sigmund, Adv. Funct. Mater., 2005, 15, 1197.
23 E. C. Le Ru, P. G. Etchegoin, J. Grand, N. Félidj, J. Aubard, G. Lévi, A. Hohenau and J. R. Krenn, Curr. Appl. Phys., 2008, 8, 467.

24 B. C. Galarreta, P. R. Norton and F. Lagugne-Labarthet, J. Phys. Chem. C, 2010, 114(47), 19952.

25 M. Bruchez, M. Moronne, P. Gin, S. Weiss and A. P. Alivisatos, Science, 1998, 281, 2013.

26 M. Han, X. Gao, J. Z. Su and S. Nie, Nat. Biotechnol., 2001, 19, 631.

27 G. Schmid, Chem. Rev., 1992, 92, 1709.

28 S. Sun, C. B. Murray, D. Weller, L. Folks and A. Moser, Science, 2000, 287, 1989.

29 G. Mie, Ann. Phys., 1908, 330, 377.

30 U. Kreibig and M. Vollmer, Optical Properties of Metal Clusters, Springer, Berlin, 1995.

31 C. F. Bohren and D. R. Huffman, Absorption and Scaterring of light by small Particles, Wiley: New York, 1983.

32 K. A. Willets and R. P. Van Duyne, Annu. Rev. Phys. Chem., 2007, 58, 267.

33 C. L. Haynes and R. P. Van Duyne, J. Phys. Chem. B, 2001, 105, 5599 .

34 L. J. Sherry, R. Jin, C. A. Mirkin, G. C. Schatz and R. P. Van Duyne, Nano Lett., 2006, 6(9), 2060.

35 L. J. Sherry, S. H. Chang, G. C. Schatz, R. P. Van Duyne, B. J. Wiley and Y. Xia, Nano Lett., 2005, 5(10), 2034.

36 W. Y. Huang, W. Qian and M. A. El-Sayed, Nano Lett., 2004, 4(9), 1741.

37 S. M. Nie and S. R. Emory, Science, 1997, 275(5303), 1102.

38 J. Zhao, A. Das, X. Y. Zhang, G. C. Schatz, S. G. Sligar and R. P. Van Duyne, J. Am. Chem. Soc., 2006, 128(34), 11004.

39 A. J. Haes, W. P. Hall, L. Chang, W. L. Klein and R. P. Van Duyne, Nano Lett., 2004, 4(6), 1029.

40 J. A. Dieringer, A. D. McFarland, N. C. Shah, D. A. Stuart, A. V. Whitney, C. R. Yonzon, M. A. Young, X. Y. Zhang and R. P. Van Duyne, Faraday Discuss., 2006, 132, 9.

41 W. A. Murray, J. R. Suckling and W. L. Barnes, Nano Lett., 2006, 6 (8), 1772.

42 C. L. Nehl, H. W. Liao and J. H. Hafner, Nano Lett., 2006, 6(4), 683.

43 A. Kosiorek, Optical, Magnetical and Structural Properties of Periodic Metallic Nanostructures, PhD Thesis, Technical University of Poznań, 2007.

44 K. Kolwas, Appl. Phys. B: Lasers Opt., 1998, 66, 467.

45 C. Bréchignac and J. P. Connerade, J. Phys. B: At., Mol. Opt. Phys., 1994, 27, 3795.

46 Y. Peng, C. Marcoux, P. Patoka, M. Hilgendorff, M. Giersig and K. Kempa, Appl. Phys. Lett., 2010, 96, 133104, and references within.

47 X. Wang and K. Kempa, Phys. Rev. B: Condens. Matter Mater. Phys., 2005, 71, 233101.

48 H. A. Bethe, Phys. Rev., 1994, 66, 163.

49 H. Levine and J. Schwinger, Phys. Rev., 1948, 74, 958.

50 C. J. Bouwkamp, Philips Res. Rep., 1950, 5, 321; C. J. Bouwkamp, Rep. Prog. Phys., 1954, 17, 35.

51 U. Dürig, D. W. Pohl and F. J. Rohner, J. Appl. Phys., 1986, 59, 3318.

52 C. L. Andrews, Phys. Rev., 1947, 71, 777.

53 G. Kolb, K. Karrai and G. Abstreiter, Appl. Phys. Lett., 1994, 65, 3090.

54 G. Ctistis, O. Schimek, P. Fumagalli and J. J. Paggel, J. Appl. Phys, 2006, 99, 014505.

55 A. Krishnan, T. Thio, T. J. Kim, H. J. Lezec, T. W. Ebbesen, P. A. Wolff, J. Pendry, L. Martin-Moreno and F. Garcia-Vidal, Opt. Commun., 2001, 200, 1.

56 T. W. Ebbesen, H. J. Lezec, H. F. Ghaemi, T. Thio and P. A. Wolff, Nature, 1998, 391, 667.

57 H. F. Ghaemi, T. Thio, D. E. Grupp, T. W. Ebbesen and H. J. Lezec, Phys. Rev. B: Condens. Matter, 1998, 58, 6779.

58 T. Thio, K. M. Pellerin, R. A. Linke, T. W. Ebbesen and H. J. Lezec, Opt. Lett., 2001, 26, 1972.

59 H. J. Lezec and T. Thio, Opt. Express, 2004, 12(16), 3629.

60 D. Pacifici, H. J. Lezec, H. A. Atwater and J. Weiner, Phys. Rev. B: Condens. Matter Mater. Phys., 2008, 77, 115411.

61 C. Brechignac, P. Houdy and M. Lahmani, Nanomaterials and Nanochemistry, Springer, Berlin, 2007.

62 D. Ricard, Optical Nonlinearities of Composite Materials: etc. in Nonlinear Optical Materials: Principles and Applications, (Proceedings of the International School of Physics), Varenna, 1993.

63 T. J. Kim, T. Thio, T. W. Ebbesen, D. E. Grupp and H. J. Lezec, Opt. Lett., 1999, 24, 256. 
64 M. Sarrazin, J.-P. Vigneron and J.-M. Vigoureux, Phys. Rev. B: Condens. Matter, 2003, 67, 085415.

65 C. Genet, M. P. van Exter and J. P. Woerdman, Opt. Commun., 2003 , 225, 331.

66 N. Bonod, S. Enoch, L. Li, E. Popov and M. Neviere, Opt. Express, $2003,11,482$.

67 A. Degiron, H. J. Lezec, W. L. Barnes and T. W. Ebbesen, Appl. Phys. Lett., 2002, 81, 4327.

68 B. Segall, Phys. Rev., 1961, 124, 1797; D. Brust, Solid State Commun., $1970,8,413$.

69 C. Guo, G. Rodriguez, A. Lobad and A. J. Taylor, Phys. Rev. Lett., 2000, 84, 4493 .

70 H. Ehrenreich, J. R. Phillipp and B. Segall, Phys. Rev., 1963, 132, 1918.

71 G. Ctistis, E. Papaioannou, P. Patoka, J. Gutek, P. Fumagalli and M. Giersig, Nano Lett., 2009, 9(1), 1.
72 B. Hecht, H. Bielefeldt, L. Novotny, Y. Inouye and D. W. Pohl, Phys. Rev. Lett., 1996, 77, 1889.

73 A. Bouhelier, Th. Huser, J. M. Freyland, H. J. Guntherodt and D. W. Pohl, J. Microsc., 1999, 194, 571.

74 C. Sönnichsen, A. C. Duch, G. Steininger, M. Koch and G. von Plessen, Appl. Phys. Lett., 2000, 76, 140.

75 A. Taflove, Computational Electrodynamics: The Finite-Difference Time-Domain Method, 2nd ed., Artech House: Norwood, MA, 2000.

76 G. Ctistis, P. Patoka, X. Wang, K. Kempa and M. Giersig, Nano Lett., 2007, 7(9), 2926.

77 E. Th. Papaioannou, V. Kapaklis, P. Patoka, M. Giersig, P. Fumagalli, A. Garcia-Martin, E. Ferreiro-Vila and G. Ctistis, Phys. Rev. B: Condens. Matter Mater. Phys., 2010, 81, 054424.

78 J. F. Torrado, E. Th. Papaioannou, G. Ctistis, P. Patoka, M. Giersig, G. Armelles and A. Garcia-Martin, Phys. Status Solidi RRL, 2010, 4 (10), 271. 